\begin{tabular}{lll} 
Abstract P159 Table 1 & & \\
\hline Level of Injury & Number & $\%$ \\
\hline C1-3 & 10 & $23 \%$ \\
C4-5 & 23 & $54 \%$ \\
$>C 5$ & 10 & $23 \%$ \\
\hline
\end{tabular}

Introduction Difficult and slow weaning of ventilated patient will have been observed by staff in many critical care units ${ }^{1}$. A National Association for Medical Direction of Respiratory Care (NAMDRC) Consensus Conference suggested that 20\% of such patients had neurological disease ${ }^{2}$.

The incidence of respiratory failure following acute cervical spinal cord injury (ASCI) ranges between $22.6 \%$ and 57\% and the average time to wean from ventilator support was found to be 36 days. Weaning for such patients should therefore take place in an intermediate care facility and be slow paced ${ }^{2}$. Previous data from our unit did suggest a successful wean in about $70 \%$ of patients admitted to this regional spinal injury unit ${ }^{1}$. We therefore wanted to review our recent results (Nov 2009 - Nov 2012) with previous standards.

Methods We performed a retrospective review of all patients admitted from November 2009 to November 2012 for respiratory weans following spinal cord insult.

Results 43 patients ( 35 male and 8 female) were admitted to the spinal critical care unit for weaning (14.33 patients per year). Average age was 54.7 years for male and 55.4 years for females. The level of injury is illustrated in the table below:

Of the 43 patients, 35 were successfully weaned; the rest were either partially weaned or not weaned.

7 of the 10 Level C1-3 injury patients were not weaned or were only partially weaned. The remaining 3 patients of Level C1-3 injury (incomplete) were weaned. 1 patient with Level C6 injury was only partially weaned but was 77 years of age.

Conclusions Review of the period from 2009-2012 is very encouraging, suggesting weaning success in line with national and international centres. Further reviews will focus on duration of wean and the effect of co-morbidities and age on the weaning outcome. Further attention needs to focus on quality of life in the weaned and not weaned patient group.

\section{REFERENCES}

1 Watt I et al. Brit I Intensive Care AUTUMN/ 2008;95-102

2 Maclntyre NR et al. Chest 2005:128:3937-3954

increased pulmonary vascular resistance, right ventricular failure and death. Survival is strongly linked to functional class with patients persisting in WHO class IV surviving less than one year. Such patients commonly require repeated hospital admissions with intractable symptoms due to right heart failure. Although specialist palliative care involvement is recommended in current guidelines for the management of $\mathrm{PAH}$, no formal recommendations exist presently to guide clinicians on timing of referral.

The aim of this study was to outline current practice in this area and define the potential workload and role of specialist palliative care services.

Methods Data was collected retrospectively for all patients within our national PAH service who died over a one year period (June 2013-June 2014). We specifically looked at timing of referral and involvement of palliative care specialists, WHO functional class, clinical course prior to death and prognostic indicators of deterioration.

Suitable patients were identified from the PAH and palliative care databases. Patient notes were reviewed to identify WHO class, clinical course prior to death and documented evidence of specialist palliative care involvement.

Results

- 31 patients were identified; (14 male, 17 female; 19 (61\%) WHO IV, 9 (29\%) WHO III, 3 (10\%) WHO II).

- Only 11 (35\%) had documented evidence of specialist palliative care involvement.

- $7(22 \%)$ received input whilst in hospital, $4(13 \%)$ in the community.

Conclusions The majority of our patients did not receive specialist palliative care support during the final stages of their disease. Whilst the majority (61\%) of patients were functional class IV prior to death, 39\% were functional class II or III. Progressive deterioration and increased burden of symptoms over time preceding death were commonly noted. Whilst the specialist PAH nurses and clinicians offer palliative care and support, our data suggests that a review of the timing, organisation and documentation of referral to specialist palliative care services requires consideration.

\title{
P161 ASSESSMENT OF AGE-ADJUSTED D-DIMER CUT-OFF VALUES IN INVESTIGATING VENOUS THROMBOEMBOLISM IN OLDER PATIENTS: A RETROSPECTIVE ANALYSIS
}

SSM Lau, GEJ Murphy. Southern General Hospital, Glasgow, UK

10.1136/thoraxjnl-2014-206260.290

\section{Pulmonary arterial hypertension: diagnosis, management and outcomes}

\section{P160 THE ROLE OF SPECIALIST PALLIATIVE CARE SERVICES IN THE MANAGEMENT OF PATIENTS WITH PULMONARY ARTERIAL HYPERTENSION; A REVIEW OF CURRENT PRACTICE}

SC Woolcock, J De Soyza, R Crackett, M Day, AJ Fisher, J Lordan, G MacGowan, PA Corris. National Pulmonary Hypertension Service (Newcastle), Institute of Cellular Medicine, Newcastle University and the NUTH NHS Foundation Trust, Newcastle Upon Tyne, UK

10.1136/thoraxjnl-2014-206260.289

Introduction and objectives Pulmonary Arterial Hypertension $(\mathrm{PAH})$ is a severe, progressive condition characterised by
Introduction and objectives The specificity of D-dimer testing in detecting venous thromboembolism (VTE) appears to fall in older patients as D-dimer concentrations increase with age. There has been interest in the use of age-adjusted D-dimer cutoff values to increase the specificity in older patients. The objective of this study was to review the diagnostic accuracy of Ddimer testing in older patients with suspected VTE in our population, comparing conventional and age-adjusted D-dimer cut-off values.

Methods A retrospective study from a large teaching hospital in the UK was undertaken. 389 data episodes were compiled from suspected VTE presentations during two months in 2013. Patients were assessed using a combination of clinical probability scores and D-dimer measurement (D-dimer HS assay, Instrumentation Laboratories). Conventional $(230 \mathrm{ng} / \mathrm{ml})$ and age-adjusted 
Abstract P161 Table 1 Characteristics of conventional and ageadjusted D-dimer cut-off values in patients $\geq 50$ years old

\begin{tabular}{llll}
\hline & Conventional & Age $\times$ 10-adjusted & Age $\times$ 3-adjusted \\
\hline Sensitivity (\%) & 100 & 76.9 & 100 \\
Specificity (\%) & 53.7 & 84.7 & 47.7 \\
Positive Predictive Value (\%) & 11.5 & 23.3 & 10.3 \\
Negative Predictive Value (\%) & 100 & 98.4 & 100 \\
\hline
\end{tabular}

(age $\times 10 \mathrm{ng} / \mathrm{ml}$ ) cut-off values were applied to patients $\geq 50$ years, and specificity and sensitivity were calculated.

Results (Table 1) Of the 389 presentations, 229 (58.9\%) were from patients aged $\geq 50$ years. $13(11.5 \%)$ patients with positive D-dimers using the conventional cut-off, had VTE as confirmed by imaging tests. The sensitivity of the conventional D-dimer cut-off value was $100 \%$ in this older cohort, with a specificity of $53.7 \%$. The age $\mathrm{x} 10$-adjusted cut-off improved specificity to 84.7\%; however sensitivity was markedly reduced to $76.9 \%$, with 3 patients $(23.1 \%)$ with non-high clinical probability of VTE missed. Further analysis suggested that an age-adjusted cutoff factor of x3 would maintain sensitivity at 100\%; however specificity was only $47.7 \%$.

Conclusions We have identified that an age-adjusted cut-off factor of $\mathrm{x} 10$ significantly increased D-dimer specificity in older patients; however the sensitivity of this test was unacceptably compromised. A cut-off factor of $\mathrm{x} 3$ maintained sensitivity, but specificity was unsatisfactory compared to conventional values, although still higher than in most published series. We conclude that we cannot use an age-adjusted cut-off of x10 in our $\geq 50$ year old population using this assay. Further work is required to identify an appropriate cut-off, concentrating on the $>75$ year old patients only. This would help to reduce the number of unnecessary tests and anxiety in this vulnerable group of patients. approximately $15 \mathrm{mSv}$, equivalent to 750 chest radiographs. ${ }^{1}$ In addition, detection of other pathology by CTPA often has minimal clinical impact. ${ }^{2}$

Aims To investigate our compliance with NICE guidelines in ordering CTPAs, and whether detecting alternative diagnoses justifies their use.

Methods This prospective study, in a London teaching hospital, reviewed data in all medical and oncology patients who had a CTPA from January to February 2014. Clinical diagnoses and risk scores were recorded according to national guidelines.

Results A CTPA was carried out on 91 patients (63\% female); 20 had confirmed PE (22\%). In 50 (55\%), guidelines were not followed: 43 did not have D-dimers, of these 15 (35\%) had cancer. Of those with PE, 35\% were detected despite low Wells Scores $(\mathrm{n}=7)$.

In $47(52 \%)$ alternative diagnoses made on CTPA accounted for the presenting symptoms: 18 diagnoses were newly made on CTPA but only 13 led to a change in management. Incidental findings requiring following were made in 9.

Conclusions PEs remain difficult to diagnose. Clinical skills may not be accurate; our detection rate was 22\%. In 55\% NICE guidelines were not followed. In 15 this was due to d dimers not being performed in patients with cancer. The testing of $d$ dimers is not routinely performed in our trust in patients with cancer, due to reduced clinical usefulness. A better scoring system may be required especially in cancer patients.

Alternative diagnoses made on CTPA do not appear to alter management in the majority, suggesting that they should not be used to make other diagnoses. More research is required in diagnosing PE to minimise radiation and contrast risks, and ensure CTPAs are of maximum clinical benefit.

\section{REFERENCES}

1 Davies et al, BMJ 2011;342:d947

2 van ES et al, Chest 2013;144(6):1893-9

\section{P163 ACCURACY OF INFLAMMATORY MARKERS TO DISTINGUISH BETWEEN PNEUMONIA AND PULMONARY EMBOLISM IN ACUTE SETTINGS}

${ }^{1} \mathrm{~L}$ Chishimba, ${ }^{2} \mathrm{~F}$ Iqbal, ${ }^{2} \mathrm{~A}$ Kasule, ${ }^{2} \mathrm{~A}$ Joynson, ${ }^{2} \mathrm{~J}$ Williams, ${ }^{3} \mathrm{~S}$ Argawal, ${ }^{1} \mathrm{I}$ Hussain. ${ }^{1}$ The University of Manchester, Manchester, UK; ${ }^{2}$ Keele University, Stoke on Trent, UK; ${ }^{3}$ University Hospital of North Staffordshire, Stoke on Trent, UK

\subsection{6/thoraxjnl-2014-206260.292}

Backgroud In emergency settings, the presence of significant rise in inflammatory markers in patients with acute respiratory symptoms suggest an alternative diagnosis rather than pulmonary embolism (PE), hence reduces the clinical probability of PE and the need for CTPA/VQ. However, it is increasingly recognised

\begin{tabular}{lllll} 
Abstract P163 Table 1 & Comparison of inflammatory markers and d-dimer levels between PE, CAP and ARTI & & \\
\hline Characteristic & PE $(\mathbf{n}=167)$ & CAP ( $\mathbf{n}=58)$ & ARTI $(\mathbf{n}=63)$ & p-value \\
\hline Gender, male (\%) & $84(50.3)$ & $31(53.4)$ & $40(63.5)$ & 0.358 \\
Age (yrs), mean (sd) & $66.9(16.6)$ & $73.4(17)$ & $63(23)$ & 0.016 \\
Hospital stay (days), median (range) & $5.31(0-8)$ & $7.08(0-34)$ & $1.51(0-29)$ & $<0.0001$ \\
30 day mortality, $n$ (\%) & $7(4.2)$ & $16(27.6)$ & $9(14.3)$ & $<0.0001$ \\
CRP (mg/l), median (range) & $67.3(4-412)$ & $88.9(12-417)$ & $68.9(9-284)$ & 0.322 \\
WCC (g/l), mean (sd) & $10.9(4.8)$ & $11.9(5.2)$ & $12.17(5.9)$ & 0.2 \\
D.dimer (ng/ml), median (range) & $1000(255-1000)$ & $513(150-1000)$ & $170(100-1000)$ & $<0.0001$ \\
Positive d-dimer (\%) & $123(96.9)$ & $8(53.3)$ & $3(18.8)$ & $<0.0001$ \\
\hline
\end{tabular}

WCC=white cell count, $C R P=C$-reactive protein, Acute $L R T=$ symptoms of acute chest infection with normal $C X R$. Normal range: WCC $=4-11 \mathrm{~g} / \mathrm{l} ; \mathrm{D}-\mathrm{dimer}=(\mathrm{mg} / \mathrm{l}) ; \mathrm{CRP}=0-5 \mathrm{mg} / \mathrm{l}$ 